\title{
BIOINFORMATICS APPROACHES TO UNDERSTAND GENE LOOPING IN THE HUMAN GENOME
}

\author{
Sudheer Menon \\ Department of Bioinformatics, Bharathiar University
}

Article DOI: $\underline{\text { https://doi.org/10.36713/epra7666 }}$

DOI No: 10.36713/epra7666

\begin{abstract}
This paper reviews up to date Bioinformatics Approaches to Understand Gene Looping in the Human Genome. Bioinformatics is used to study the sequences of biological molecules. It generally points out to genes, DNA, RNA, or protein, and is especially functional in analogizing genes and other protein sequences. You can believe in bioinformatics. Basically, the linguistics Bioinformatics uses computer programs for various applications, involving deliberate gene and protein functions. The beginning of the human genome project in 1990 and was completed in 2003. The Human Genome Project gave a prime improvement for the progress of bioinformatics. The (HGP) was organized by the National Institutes of Health and the U.S. Department of Energy. Without the interpretation given via bioinformatics, the information obtained from the HGP is not very functional. This page describes HGP bioinformatics research. Informatics is the formation, exploration, and function of databases. Main aim was to find the total set of human genes and make them available for more biological study and discover the total sequence of $D N A$ bases in the human genome. A total and the correct sequence of the 3 billion DNA base pairs create the human genome and search all approximate 20,000 to 25,000 human genes. The genomes sequence of organisms that are main to medical research. To begin new tools to apply and inspect the data and to assemble this information broadly obtainable.DNA sequencing manufactures a sequence that is particularly a hundred bases long. Gene sequences manufacture thousands of bases. To study genes, small intersecting sequences set up long DNA sequences. Loops can clump associated genes into separate transcriptional axis chromatin from neighboring domains. Gene loops in yeast juxtapose promoter-terminator regions. Here we outline gene loops' finding, the looping need proteins, and transcription by $R N A$ polymerase II is by gen looping
\end{abstract}

\section{INTRODUCTION}

An unmatched means of biological data has originated from the human genome project. The numerous request for inspection and explanation of these data is made possible by the developing science of bioinformatics. The definition of Bioinformatics the implementation of tools and assessment to the catch and description of biological data. It is an associative area, which tackles computer science, mathematics, and biology. Bioinformatics is necessary for the control of data in biology and medicine. This paper related to bioinformatician's primary instruments and debated how they are cast-off to explain biological data. The possible impersonal implementation of these data in drug finding and growth. In the human body, each cell is coded by the same DNA sequence of genetic information. A series of DNA-binding proteins arrange transcriptional regulation of gene expression. TF binding mostly happens at clumps of DNA organization sequences called 'promoters' and 'enhancers.' It is hugely base on the unique approachability and pursuit of the primary DNA sequence filled into chromatin. Naturally, chromatin boatyard various concretion levels and transcriptionally 


\section{SJIF Impact Factor 2021: 8.013| ISI I.F.Value:1.241| Journal DOI: 10.36713/epra2016 ISSN: 2455-7838(Online) EPRA International Journal of Research and Development (IJRD)

active regions cluster an additional open structure. The receptivity of the chromatin is also base on the gene verdancy of the area. The chromatin organization's first level is the nucleosome and of an octamer of histones $(\mathrm{H} 2 \mathrm{~A}, \mathrm{H} 2 \mathrm{~B}, \mathrm{H} 3$, and $\mathrm{H} 4)$ throughout which DNA is enclosed. Histone improvement may change the association between histone and DNA or act as a tie-up policy for proteins or protein complexes that may moderate chromatin improvement.

\section{BIOINFORMATICS AND ITS EFFECT ON GENOMICS}

The complete human genome had been plotted due to the struggle of the worldwide human genome project and a private genomics company. Although, in the present day, the scientific world has observed the consumption of complete genome sequences of numerous other organisms. The inspection of the proceeds of genomic sequence data and the human genome project is a turning point for bioinformatics. A hardback strategy for spontaneous sequencing of the full genome use to sequence Haemophilus influenza's genome in 1995. This was the whole genome of any unbound living organism to be sequenced. The arrangement and interpretation of the first eukaryotic genome that of Saccharomyces cerevisiae (a yeast) was go along by other eukaryotic species such as Caenorhabditis elegans, Drosophila melanogaster, many species sequences, encompass non-human primates, pufferfish, mouse, rat, approaching completion sequencing enterprise The knowledge to acquire from these sequence data will have substantial involvement for our awareness of biology and medicine. As a result of comparative genomics. We will be efficient to not only detect each human gene but also completely acknowledge its function.

\section{BIOINFORMATIC TOOLS}

The fundamental tools of bioinformatics are computer software programs and the internet. The main occupation is sequence inspection of DNA and proteins using different programs and databases accessible worldwide. Every person using bioinformatics tools can openly find out the composition of biology. This does not suggest that all can sustain control and inspection of raw genomic data. Bioinformatics is a developed regulation, and specialist bioinformaticians now utilize composite software programs for recovery, categorize investigation, DNA, and protein sequence data. Huge trading pursuits such as pharmaceutical companies hire bioinformaticians to carry out and sustain the huge scale and composite bioinformatic required of these industries... Behind a basic accession and inspection of simple data, the independent investigator would surely require an exterior bioinformatic guide for any complex examination.

The extension of bioinformatics has been a worldwide journey, generating computer networks that have permitted uncomplicated ingress to biological data and authorized software programs for straightforward examination. Many international projects lineup as long as gene and protein databases are accessible openly to the entire scientific community along with the internet.

\section{BIOINFORMATIC ANALYSIS}

The accelerated volume of data from the genome projects has constrained computer databases that characterize quick immersion and workable setup; because of the various essence of proceeding data, no one extensive database exists to acquire all this detail. However, an increased number of databases that hold a beneficent point for impersonal and investigator are obtainable. The topic given by most of these databases is for nothing to academics. However, some sites need contribution, and industrial customers repay a license fee for specific areas. Sample reach from locations given an extensive explanation of impersonal disarranged, record disease responsiveness genetic mutations and polymorphisms, to those authorize a explore for disease genes given a DNA sequence (box). The uncomplicated way to recognize databases is by exploring bioinformatics tools, and databases are frequently cast-off search engines. Many other ways to identify bioinformatic origin are always database connection and explore indexes given by one of the vital common databases. For example, the National Center for Biotechnology Information imparts the Entrez Browser, an integrated database system that allows incorporating DNA and protein sequence databases. The European Bioinformatics Institute records gene and protein data from genome studies of all organisms, expressions Ensembl provides, and keeps up mechanized notation on eukaryotic genomes. The features and accuracy of databases differ; some of the more significant known and more accepted ones.

One of the uncomplicated and greater familiar explore tools is called BLAST. This algorithm software is efficient for exploring databases for genes. It allows humans or other organisms to resemble hundreds or thousands of sequences of unspecified DNA or amino acid sequence. Databases of efficient sequences make use of to recognize similar arrangements, which may be homologous. Homology suggested that sequence may be associate with disagreement from a usual forefather 
or division of usual useful sense. When a database is a search with a recent purposive sequence, regional arrangement happens between the inquiry sequence and alike sequence in the database. The outcome of the hunt is settle in the arrangement of preference based on the most astonishing resemblance. The sequence with the most incredible development in the database of normal genes is the homolog.

\section{FUNCTIONAL GENOMICS}

The fulfillment of the first draft of the human genome, genomic information, is the applicability of available genomic appointment - the study of genes, the role of the proteins, and their resulting proteins.

Inspection and explanation of biological data suppose information at the level of the proteome and the transcriptome but not only at the level of the genome Proteomics is the inspection of the entire amount of proteins convey by a cell, and transcriptomics involves to the assessment of the messenger RNA transcripts provide by a cell. DNA technology incorporates genotyping and DNA sequencing and establishes the declaration amount of genes. Gene expression arrangement permits concurrent inspection of the messenger RNA expression of genes. Bioinformatic protein investigation extract to interpret protein and twodimensional electrophoresis databases. Bioinformatics is the projection structure of protein After detachment, recognition, and delineation. Biologists also utilize bioinformatics to pick up the vast and composite data to make three-dimensional models of molecules from nuclear magnetic resonance and electron microscopy.

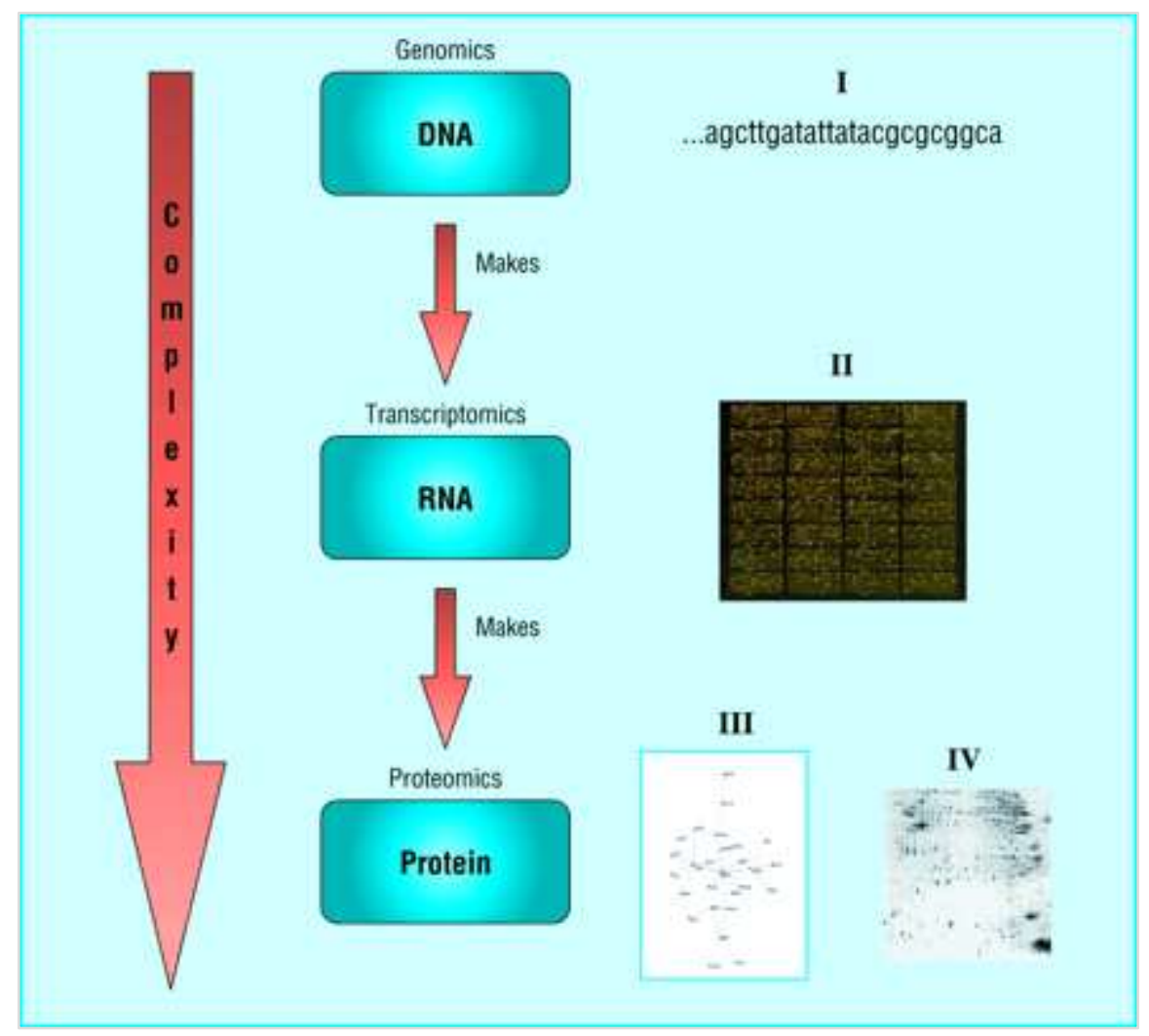

The graphic diagram symbolizes the complication of data processing of the genome. Inspection and explanation of biological data information total protein content and total genetic content and total messenger RNA content of the cell. The figure numbered I-IV to the accurate diagram 


\section{SJIF Impact Factor 2021: 8.013| ISI I.F.Value:1.241| Journal DOI: 10.36713/epra2016 ISSN: 2455-7838(Online) EPRA International Journal of Research and Development (IJRD)

\section{ANALYSIS OF THE FRACTION OF CTCF AND COHESIN MOLECULES REQUIRE IN LOOPING}

CTCF and cohesin both have purpose after modulate chromatin looping, and the main question fragment of chromatin-bound CTCF and cohesin sites are required in chromatin looping. The number of engaged binding sites are roughly ChIP-Seq and recognized as top remarkable above a behind the scenes beginning. assessment, a spectrum of binding enhancement is all the time and summit profession requires a bit of unpredictable discretization step. utilizing MACS2 and quality framework. We call 68,077 CTCF ChIP-Seq tops in wild-type mESCs and a matching number in Halo-mCTCF hammer cells (C59; see Supplementary file 1 for full details). In addition for cohesin we notice 33,434 ChIP-Seq spires of which $97 \%$ of the summit intersection with a CTCF top. consequently the cohesin top seems to be a subset of CTCF top and there seem to be remarkable cohesin binding at more other CTCF tops, albeit under the summit occupation start.

What fragment of $\mathrm{CTCF} /$ cohesin sites are provided in looping? About calling top utilizing ChIPSeq data, spirals are also normally yell by fragment $\mathrm{Hi}-$ $\mathrm{C}$ data and seem as corner-peaks in the Hi-C interconnection matrix. Various groups have utilized various approaches and $\mathrm{Hi}-\mathrm{C}$ data at various determination and appropriately have announced various numbers of loops (Jin et al., 2013; Rao et al., 2014; Sanyal et al., 2012). The extreme resolution Hi-C data announced to date is from Rao et al. and they report $\sim 10,000$ spiral utilizing a very firm and conventional loop-calling innovation in GM12878 cells. The alike group called considerably insufficient loops in future cell border sequenced at a bottom sequencing bowels although, utilizing a technique called Aggregate Peak Analysis (APA), which permits $\mathrm{Hi}-\mathrm{C}$ chart at various proposal to be resemble, Rao et $a l$. establish that the little loops were require to the bottom sequencing below slightly than an lacking of loops in cell lines. Actually, they establish that loops were mostly safeguard between various cell lines. Thus, it like the ability to call loops depends on sequencing depth and thus, it appears probable that big resolution $\mathrm{Hi}-\mathrm{C}$ data may be accessible in the future, the number of assurance loops will outstandingly overreach 10,000. Following Rao et al., almost all Hi-C loops are secure by both CTCF and cohesin. consequently, a beneath leap approximate would be that 20,000 CTCF and Cohesin ChIP-Seq sites attach loops. Nevertheless, as also spearlike out by Rao et al. and distinguishable verify by instructive current analysis by Merkenschlager and Nora, numerous loops seems to be attach by collection of $\mathrm{CTCF} /$ cohesin binding sites. consequently since more CTCF and cohesin ChIP-Seq sites can attach the alike loop, 20,000 appears to be excessively little bound. In the major content of the document, we mention this as throughout one-third of CTCF sites and as an age of consent cohesion sites. We as well observe that within the expulsion model, a remarkable fragment of cohesin that are physiography involved on chromatin that energetically proceed over the chromosome and this fragment is improbable to be plucked up by ChIP-Seq peak-calling analysis. This fragment would seem identical from cohesin crumb leap at particular loop partition in our FRAP inspection. However between cohesin molecules that endure at a particular situation for an expanded session.

\section{REFERENCES}

1. Initial sequencing and inspection of the human genome. International human genome sequencing. Nature. 2001,409:860_921.(PubMed) (Google Scholar)

2. Venter JC, Myers EW, Adams MD, Mural RJ, Li $P W$, Sutton GG, et al. The Human Genomic sequence. Science. 2001,291:1304-1351. (PubMed) (Google Scholar)

3. Fleischmann RD, Adams MD, Clayton $R A$, Kirkness EF, Kerlavage AR, et al., a random sequence of complete Genome. Science. 1995,269:496-512. (PubMed) (Google Scholar)

4. Adams MD, Gocayne JD, White O, Fraser CM, the small gene complement of Mycoplasma. science.1995,270;397-403.(PubMed) (Google Scholar)

5. Parkhill J, Garnier T, Cole ST, Harris D, Brosch R, et al. The biology of Mycobacterium tuberculosis from the whole genome sequence. Nature. 1998;393;537-544. (PubMed) (Google Scholar) 